\title{
Expanding the realm of systematic proof theory
}

\author{
Agata Ciabattoni ${ }^{1}$, Lutz Straßburger ${ }^{2}$, and Kazushige Terui ${ }^{3}$ \\ ${ }^{1}$ Technische Universtät Wien, Austria $\quad{ }^{2}$ INRIA Saclay-Île-de-France, France \\ ${ }^{3}$ RIMS, Kyoto University, Japan
}

\begin{abstract}
This paper is part of a general project of developing a systematic and algebraic proof theory for nonclassical logics. Generalizing our previous work on intuitionistic-substructural axioms and singleconclusion (hyper)sequent calculi, we define a hierarchy on Hilbert axioms in the language of classical linear logic without exponentials. We then give a systematic procedure to transform axioms up to the level $\mathcal{P}_{3}^{\prime}$ of the hierarchy into inference rules in multiple-conclusion (hyper)sequent calculi, which enjoy cut-elimination under a certain condition. This allows a systematic treatment of logics which could not be dealt with in the previous approach. Our method also works as a heuristic principle for finding appropriate rules for axioms located at levels higher than $\mathcal{P}_{3}^{\prime}$. The case study of Abelian and Łukasiewicz logic is outlined.
\end{abstract}

\section{Introduction}

Since the axiomatisation of classical propositional logic by Hilbert in 1922, such axiomatic descriptions (nowadays called Hilbert-systems) have been successfully used to introduce and characterize logics. Ever since Gentzen's seminal work it has been an important task for proof theorists to design for these logics deductive systems that admit cut-elimination. The admissibility of cut is crucial to establish important properties of corresponding logics such as consistency, decidability, conservativity, interpolation, and is also the key to make a system suitable for proof search. As designers of deductive systems could never keep pace with the speed of logicians and practitioners coming up with new logics, general tools to automate this design process and extract suitable rules out of axioms would be very desirable. Work in this direction are e.g. [7, 20, 19].

A general project of systematic and algebraic proof theory for nonclassical logics was recently launched in $[3,4]$ where Hilbert axioms in the language of full Lambek calculus FL (i.e., intuitionistic noncommutative linear logic without exponentials) have been classified into the substructural hierarchy $\left(\mathcal{P}_{n}, \mathcal{N}_{n}\right)_{n \in \mathbb{N}}$, with the aim to conquest the whole hierarchy from bottom to top. The work in [3] successfully dealt with the axioms up to level $\mathcal{N}_{2}$. It gave a procedure to transform them into structural rules in the single-conclusion sequent calculus, and algebraically proved (a stronger form of) cut-elimination for FL extended with the generated rules which satisfy the syntactic condition of acyclicity. Then, [4] expanded the realm to the level $\mathcal{P}_{3}^{\prime}$, a subclass of $\mathcal{P}_{3}$ in the commutative setting, by using the single-conclusion hypersequent calculus [2]. The aim to continue the conquer further faced a serious obstacle: As shown in [3], "strong" 
cut-elimination for a logical system $L$ implies that the class of algebras corresponding to $L$ is closed under completions, whereas certain logics beyond $\mathcal{P}_{3}^{\prime}$ do not admit closure under completions. Typical examples are Abelian logic AL [15, 16] - the logic corresponding to compact closed categories - and infinite-valued Łukasiewicz logic $\ell$, although possessing cut-free hypersequent calculi, see [14].

In this paper, we circumvent the obstacle by shifting from the intuitionistic and single-conclusion setting to the classical and multiple-conclusion one. This causes a deconstruction of the hierarchy; certain axioms which resided at high levels are brought down to lower levels, to the reach of our systematic proof theory (Section 3). Generalizing the method in [3,4], Section 4 (resp. Section 5) describe a procedure to transform any $\mathcal{N}_{2}$ axiom (resp. $\mathcal{P}_{3}^{\prime}$ axiom) into structural rules in the multiple-conclusion sequent (resp. hypersequent) calculus. The procedure is also applied to obtain logical rules for connectives defined by certain Hilbert axioms. Section 6 outlines a uniform syntactic cut-elimination procedure that works for the generated rules satisfying the acyclicity condition. This allows the systematic introduction of cut-free calculi for logics which cannot be dealt with in the single-conclusion approach, such as 3-valued Łukasiewicz logic and Nelson's logic. Our method also works as a heuristic principle for finding appropriate rules for axioms located at levels higher than $\mathcal{P}_{3}^{\prime}$. As a case study, in Section 7 we show how to semi-automatically obtain the cut-free hypersequent calculi for AL and $\ell$, that have been discovered in [14] by trial and error.

\section{Preliminaries: Sequents and Hypersequents}

We consider formulas to be generated from a set $\mathcal{V}=\{a, b, c, \ldots\}$ of propositional variables, their duals $\mathcal{V}^{\perp}=\left\{a^{\perp}, b^{\perp}, c^{\perp}, \ldots\right\}$, and the constants $\perp, 1,0$, and $\top$ via the binary connectives $\diamond, \otimes, \oplus$, and $\&::^{1}$

$$
\mathcal{F}::=\mathcal{V}\left|\mathcal{V}^{\perp}\right| \perp|1| 0|\top| \mathcal{F} \& \mathcal{F}|\mathcal{F} \otimes \mathcal{F}| \mathcal{F} \oplus \mathcal{F} \mid \mathcal{F} \& \mathcal{F}
$$

We use $A, B, C, \ldots$ to denote formulas, and we define the negation on formulas via the usual DeMorgan equalities. It follows immediately that $A^{\perp \perp}=A$ for all $A$. We write $A \multimap B$ for $A^{\perp} \& B$, and $A \multimap B$ for $(A \multimap B) \&(B \multimap A)$. For reasons that will become clear later, we will write $A_{\& 1}$ for $A \& 1$.

We will also speak about axiom (schemes) $\phi, \psi, \ldots$, which are generated by the same grammar as (1), but starting from formula variables instead of propositional variables. By some abuse of notation, we use $A, B, C, \ldots$ to denote formula variables. We call an axiom $\phi$ atomic if $\phi=A$ or $\phi=A^{\perp}$ for some formula variable $A$. By some further abuse of notation, we will use $A, B, C, \ldots$ to denote atomic axioms (positive or negative).

\footnotetext{
${ }^{1}$ We use here the notation used in the linear logic community. The table below gives the translation to the notation used in the substructural logics community.
}

$$
\begin{array}{lcccccccc}
\text { linear logic: } & \diamond & \otimes & \oplus & \& & \perp & 1 & 0 & \top \\
\text { substructural logics: } & \oplus & \cdot / \odot & \vee & \wedge & 0 & 1 & \perp & \top
\end{array}
$$




\begin{tabular}{|c|c|c|c|}
\hline ax $\overline{\mathscr{H} \mid \vdash A, A^{\perp}}$ & cut $\frac{\mathscr{H}|\vdash \Gamma, A \quad \mathscr{H}| \vdash A^{\perp}, \Delta}{\mathscr{H} \mid \vdash \Gamma, \Delta}$ & ew $\frac{\mathscr{H}}{\mathscr{H} \mid \vdash \Gamma}$ & ec $\frac{\mathscr{H}|\vdash \Gamma| \vdash \Gamma}{\mathscr{H} \mid \vdash \Gamma}$ \\
\hline $1 \overline{\mathscr{H} \mid \vdash 1}$ & $\otimes \frac{\mathscr{H}|\vdash \Gamma, A \quad \mathscr{H}| \vdash B, \Delta}{\mathscr{H} \mid \vdash \Gamma, A \otimes B, \Delta}$ & $\perp \frac{\mathscr{H} \mid \vdash \Gamma}{\mathscr{H} \mid \vdash \Gamma, \perp}$ & ஓ $\frac{\mathscr{H} \mid \vdash \Gamma, A, B}{\mathscr{H} \mid \vdash \Gamma, A \ngtr B}$ \\
\hline$\top \overline{\mathscr{H} \mid \vdash \Gamma, \top}$ & $\& \frac{\mathscr{H}|\vdash \Gamma, A \quad \mathscr{H}| \vdash \Gamma, B}{\mathscr{H} \mid \vdash \Gamma, A \& B}$ & $\oplus_{1} \frac{\mathscr{H} \mid \vdash \Gamma, A}{\mathscr{H} \mid \vdash \Gamma, A \oplus B}$ & $\oplus_{2} \frac{\mathscr{H} \mid \vdash \Gamma, B}{\mathscr{H} \mid \vdash \Gamma, A \oplus B}$ \\
\hline
\end{tabular}

Fig. 1. Hypersequent system HMALL

We write $\bigoplus_{i=1}^{n} A_{i}$, or simply $\bigoplus_{i} A_{i}$ to abbreviate $A_{1} \oplus A_{2} \oplus \cdots \oplus A_{n}$, where $\oplus_{i=1}^{n} A_{i}=0$ if $n=0$, and similarly for the other connectives.

Definition 2.1 A (single sided) sequent is a finite multiset of formulas, usually written as $\vdash A_{1}, \ldots, A_{n}$. A (single sided) hypersequent $\mathscr{H}$ is a finite multiset of sequents written as $\vdash \Gamma_{1}|\ldots| \vdash \Gamma_{n}$. The interpretation $(\vdash \Gamma)^{\mathrm{I}}$ of a sequent $\vdash \Gamma=\vdash A_{1}, \ldots, A_{n}$ is the formula $A_{1} \& \ldots \ngtr A_{n}$, and $(\vdash \Gamma)^{\mathrm{I}}=\perp$, if $n=0$. For a hypersequent $\mathscr{H}=\vdash \Gamma_{1}|\ldots| \vdash \Gamma_{n}$, we define $\mathscr{H}^{\mathrm{I}}=\left(\vdash \Gamma_{1}\right)_{\& 1}^{\mathrm{I}} \oplus \cdots \oplus\left(\vdash \Gamma_{n}\right)_{\& 1}^{\mathrm{I}}$.

Henceforth we use $\Gamma, \Delta, \Sigma, \ldots$ to denote multisets of formulas, and $\mathscr{G}, \mathscr{H}, \ldots$ to denote hypersequents. We denote by HMALL the hypersequent system shown in Figure 1. With MALL we denote the corresponding sequent system, obtained from HMALL by removing the rules ec and ew, and by dropping the hypersequent context $\mathscr{H}$ everywhere. In inference rules we will refer to $\Gamma, \Delta, \Sigma, \ldots$ as multiset variables (as opposed to the formula variables $A, B, \ldots$ ).

The notation $\vdash_{\mathrm{s}} A$ (respectively $\vdash_{\mathrm{s}} \Gamma$ or $\vdash_{\mathrm{s}} \mathscr{H}$ ) will mean that a formula $A$ (respectively a sequent $\vdash \Gamma$ or a hypersequent $\mathscr{H}$ ) is provable in the system $\mathrm{S}$.

Proposition 2.2 For any sequent $\vdash \Gamma$ and hypersequent $\mathscr{G}$, we have that

$$
\vdash_{\mathrm{HMALL}+\mathscr{G}} \Gamma \quad \text { iff } \quad \vdash_{\mathrm{MALL}+\mathscr{G} I} \Gamma .
$$

Proof For the 'if' direction, observe that $\mathscr{G}^{I}$ is derivable from $\mathscr{G}$ in HMALL. For the converse, prove by induction that $\vdash_{\text {HMALL }+\mathscr{G}} \mathscr{H}$ implies $\vdash_{\text {MALL }+\mathscr{G} I} \mathscr{H}^{I}$.

Definition 2.3 Given two sets of inference rules $S_{1}$ and $S_{2}$, we say that $S_{1}$ and $\mathrm{S}_{2}$ are equivalent iff $(\mathrm{H}) \mathrm{MALL}+\mathrm{S}_{1}$ and $(\mathrm{H}) \mathrm{MALL}+\mathrm{S}_{2}$ prove the same sequents. If $S_{1}=\{r\}$ is a singleton, we simply write $(H) M A L L+r$.

An axiom $\phi$ is a rule without premises. Thus, the definition above applies also to (sets of) axioms.

Remark 2.4 By moving to the single-sided (multiple conclusion) setting, we do not lose any expressive power of the two-sided single-conclusion setting (i.e. 


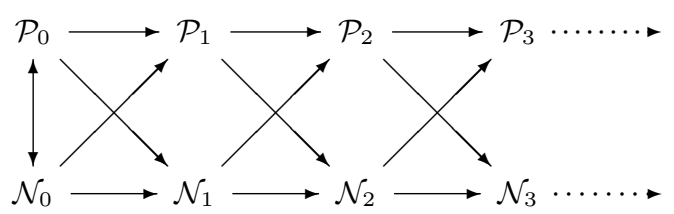

Fig. 2. The Substructural Hierarchy

involving sequents of the form $\Gamma \vdash A$ ). Indeed the latter can faithfully be embedded into the former by using left/right polarities, referring to the left and the right side of a two-sided sequent (see, e.g., [8,9] for details). We can then call a MALL formula (or axiom) intuitionistic, if it has right polarity. A MALL sequent $\vdash \Gamma$ is intuitionistic iff at most one formula in $\Gamma$ has right polarity, and all other formulas in $\Gamma$ have left polarity, and a proof in MALL is called intuitionistic if all its lines are intuitionistic sequents. We then have that a formula $A$ belongs to intuitionistic logic iff its translation $A^{c}$ into the classical language is intuitionistic in our sense. Let IMALL denote the usual two-sided sequent calculus for intuitionistic MALL (also known as full Lambek calculus with exchange, FLe), then we have $\vdash_{\text {IMALL }} \Gamma \vdash A$ iff there is an intuitionistic proof of $(\Gamma \vdash A)^{c}$ in MALL. Furthermore, If $A$ does not contain any occurrences of $\perp$ or $\top$, then $\vdash_{\text {IMALL }} \Gamma \vdash A$ iff $\vdash_{\text {MALL }}(\Gamma \vdash A)^{c}$. The reason is that MALL is a conservative extension of IMALL without $\perp$ and $\top[21]$.

\section{Substructural Hierarchy}

Following [3, 4], we define a hierarchy $\left(\mathcal{P}_{n}, \mathcal{N}_{n}\right)$ on formulas of MALL. It is based on the polarities of the connectives [1], which is also the basis for focusing and linear logic programming [17]. Recall that logical connectives of MALL can be classified into two groups: negative $(\diamond, \&, \perp$, and $\top)$ and positive $(\oplus, \otimes, 0$, and 1 ), according to the fact that their sequent calculus rules are invertible and non-invertible, respectively.

Let $\mathcal{A}$ be the set of atomic axioms. The classes $\mathcal{P}_{n}$ and $\mathcal{N}_{n}$ of positive and negative axioms are defined via the following grammar:

$$
\begin{array}{ll}
\mathcal{P}_{0}::=\mathcal{A} & \mathcal{P}_{n+1}::=\mathcal{N}_{n}\left|\mathcal{P}_{n+1} \otimes \mathcal{P}_{n+1}\right| \mathcal{P}_{n+1} \oplus \mathcal{P}_{n+1}|1| 0 \\
\mathcal{N}_{0}::=\mathcal{A} & \mathcal{N}_{n+1}::=\mathcal{P}_{n}\left|\mathcal{N}_{n+1} \& \mathcal{N}_{n+1}\right| \mathcal{N}_{n+1} \& \mathcal{N}_{n+1}|\top| \perp
\end{array}
$$

We have the following immediate observations:

Proposition 3.1 Every axiom belongs to some $\mathcal{P}_{n}$ and some $\mathcal{N}_{n}$, and for all $n$, we have $\mathcal{P}_{n} \subseteq \mathcal{P}_{n+1}$ and $\mathcal{N}_{n} \subseteq \mathcal{N}_{n+1}$. Furthermore, $A \in \mathcal{P}_{n}$ iff $A^{\perp} \in \mathcal{N}_{n}$.

Hence we have a hierarchy, called the substructural hierarchy in [3, 4], which can be depicted as in Figure 2 . 


\begin{tabular}{|c|c|c|c|}
\hline Axiom (intuitionistic and classical version) & Name & Rule & Class \\
\hline $\begin{aligned} A \multimap & 1, \perp \multimap A \\
& A \ngtr 1\end{aligned}$ & weakening & w & $\begin{array}{l}\mathcal{N}_{2} \\
\mathcal{N}_{2}\end{array}$ \\
\hline $\begin{array}{c}A \multimap A \otimes A \\
A^{\perp} 8(A \otimes A)\end{array}$ & contraction & c & $\begin{array}{l}\mathcal{N}_{2} \\
\mathcal{N}_{2} \\
\end{array}$ \\
\hline $\begin{array}{c}A \oplus(A \multimap \perp) \\
A \oplus A^{\perp}\end{array}$ & excluded middle & em & $\begin{array}{l}\mathcal{P}_{2} \\
\mathcal{P}_{1}\end{array}$ \\
\hline $\begin{array}{ll}\mathrm{i}: & (A \multimap B)_{\& 1} \oplus(B \multimap A)_{\& 1} \\
\text { c: } & \left(A^{\perp} \diamond B\right)_{\& 1} \oplus\left(B^{\perp} \diamond A\right)_{\& 1}\end{array}$ & linearity & com & $\begin{array}{l}\mathcal{P}_{3}^{\prime} \\
\mathcal{P}_{3}^{\prime}\end{array}$ \\
\hline $\begin{array}{l}\text { i: } \quad\left(\left(A^{\otimes 2} \multimap B\right) \&\left((B \multimap \perp)^{\otimes 2} \multimap(A \multimap \perp)\right)\right) \multimap(A \multimap B) \\
\text { c: } \quad((A \otimes A \otimes B) \oplus(B \otimes B \otimes A)) \& A^{\perp} \ngtr B^{\perp}\end{array}$ & Nelson axiom & nel & $\begin{array}{l}\mathcal{N}_{3} \\
\mathcal{N}_{2}\end{array}$ \\
\hline $\begin{array}{lc}\text { i: } & A \oplus\left(A^{\otimes n} \multimap \perp\right) \\
\text { c: } & A \oplus\left(A^{\perp}\right)^{8 n} \\
\end{array}$ & $n$-excluded middle & $n$-em & $\begin{array}{l}\mathcal{P}_{2} \\
\mathcal{P}_{2}\end{array}$ \\
\hline
\end{tabular}

Fig. 3. Axioms and their level in the substructural hierarchy

Remark 3.2 In [4] the hierarchy was defined on formulas of IMALL as follows:

$$
\begin{aligned}
\mathcal{P}_{0}^{i}::=\mathcal{A} & \mathcal{P}_{n+1}^{i}::=\mathcal{N}_{n}^{i}\left|\mathcal{P}_{n+1}^{i} \otimes \mathcal{P}_{n+1}^{i}\right| \mathcal{P}_{n+1}^{i} \oplus \mathcal{P}_{n+1}^{i}|1| 0 \\
\mathcal{N}_{0}^{i}::=\mathcal{A} & \mathcal{N}_{n+1}^{i}::=\mathcal{P}_{n}^{i}\left|\mathcal{N}_{n+1}^{i} \& \mathcal{N}_{n+1}^{i}\right| \mathcal{P}_{n+1}^{i} \multimap \mathcal{N}_{n+1}^{i}|\top| \perp,
\end{aligned}
$$

where $\mathcal{A}$ ranges over positive atomic axioms (without negation). It follows from Remark 2.4 that the two hierarchies coincide: $\phi \in \mathcal{P}_{n}^{i}$ iff $\phi^{c} \in \mathcal{P}_{n}$, and $\phi \in \mathcal{N}_{n}^{i}$ iff $\phi^{c} \in \mathcal{N}_{n}$.

Figure 3 shows some examples of axioms and their class, and Figure 4 shows the corresponding structural rules. How they are obtained will be explained in the course of this paper. Observe we can have the following situation: For a certain intuitionistic axiom $\phi$ there is an axiom $\phi^{\prime}$ located in a lower class of the hierarchy such that $\phi^{\prime}$ is not intuitionistic and $\vdash_{\text {MALL }} \phi \circ \circ \phi^{\prime}$. The use of the classical language also simplifies the following statement, established in [4], which will be used to make syntactic transformations of axioms.

Proposition 3.3 Every axiom $\phi \in \mathcal{P}_{n+1}$ is equivalent to an axiom of the form $\oplus_{i}\left(\otimes_{j} \psi_{i, j}\right)$ where $\psi_{i, j} \in \mathcal{N}_{n}$ for each $i, j$. And every axiom $\phi \in \mathcal{N}_{n+1}$ is equivalent to an axiom of the form $\&_{i}\left(\bigotimes_{j} \psi_{i, j}\right)$ where $\psi_{i, j} \in \mathcal{P}_{n}$ for each $i, j$.

Definition 3.4 An axiom $\phi$ is called $\mathcal{N}_{2}$-normal if it is of the shape

$$
\phi=8_{k}\left(\oplus_{i}\left(\otimes_{j} A_{k, i, j}\right)\right) \quad \text { where each } A_{k, i, j} \text { is atomic. }
$$

It follows immediately from Proposition 3.3 that any $\mathcal{N}_{2}$-axiom can be transformed into a finite conjunction (\&) of $\mathcal{N}_{2}$-normal axioms. As in [4], for dealing with systems having no weakening, we consider a subclass of $\mathcal{P}_{3}$ that we call $\mathcal{P}_{3}^{\prime}$, which is generated by the grammar:

$$
\mathcal{P}_{3}^{\prime}::=\mathcal{N}_{2} \& 1\left|\mathcal{P}_{3}^{\prime} \otimes \mathcal{P}_{3}^{\prime}\right| \mathcal{P}_{3}^{\prime} \oplus \mathcal{P}_{3}^{\prime}|1| 0
$$




$$
\begin{gathered}
\mathrm{w} \frac{\vdash \Gamma}{\vdash \Gamma, \Delta} \quad \mathrm{c} \frac{\vdash \Gamma, \Delta, \Delta}{\vdash \Gamma, \Delta} \quad \text { em } \frac{\vdash \Gamma, \Gamma}{\vdash \Gamma} \quad \operatorname{com} \frac{\vdash \mathscr{H}|\Gamma, \Theta \quad \vdash \mathscr{H}| \Sigma, \Delta}{\mathscr{H}|\vdash \Gamma, \Delta| \vdash \Sigma, \Theta} \\
\quad \text { nel } \frac{\vdash \Gamma, \Sigma, \Sigma, \Delta \quad \vdash \Gamma, \Sigma, \Delta, \Delta}{\vdash \Gamma, \Delta, \Sigma} \quad n \text {-em } \frac{\mathscr{H}\left|\vdash \Gamma, \Sigma_{1} \quad \cdots \quad \mathscr{H}\right| \vdash \Gamma, \Sigma_{n}}{\mathscr{H}|\vdash \Gamma| \vdash \Sigma_{1}, \ldots, \Sigma_{n}}
\end{gathered}
$$

Fig. 4. Rules generated from axioms in Figure 3

Lemma 3.5 The set $\left\{\phi_{\& 1} \oplus \xi, \psi_{\& 1} \oplus \xi\right\}$ is equivalent (in the sense of Definition 2.3) to $\left(\phi_{\& 1} \otimes \psi_{\& 1}\right) \oplus \xi$ as well as to $(\phi \& \psi)_{\& 1} \oplus \xi$, for any $\phi, \psi$, and $\xi$.

Proof Follows from provability in MALL of $\left(A_{\& 1} \oplus C\right) \otimes\left(B_{\& 1} \oplus C\right)-\left(A_{\& 1} \otimes B_{\& 1}\right) \oplus C$ and $\left(A_{\& 1} \otimes B_{\& 1}\right) \oplus C \multimap(A \& B)_{\& 1} \oplus C$, for all formulas $A, B$, and $C$.

Proposition 3.6 Every axiom $\phi \in \mathcal{P}_{3}^{\prime}$ is equivalent to a finite set $\left\{\psi_{1}, \ldots, \psi_{n}\right\}$ of axioms such that $\psi_{i}=\bigoplus_{j=1}^{m_{i}}\left(\xi_{i, j}\right)_{\& 1}$ where $\xi_{i, j}$ is $\mathcal{N}_{2}$-normal for all $i, j$.

Proof We have $\phi \circ \bigoplus_{j} \otimes_{k}\left(\&_{l} \xi_{j, k, l}\right)_{\& 1}$ where $\xi_{j, k, l}$ is $\mathcal{N}_{2}$-normal, so that we can apply Lemma 3.5.

\section{From $\mathcal{N}_{2}$-axioms to Sequent Rules}

In this section we provide an algorithm for transforming $\mathcal{N}_{2}$ axioms into equivalent sequent calculus rules. Our algorithm extends and simplifies the one introduced in [4] (and in [3], for the noncommutative case) for axioms in $\mathcal{N}_{2}^{i}$ and structural rules. A suitable modification of the procedure also enables us to extend the result to logical rules.

Lemma 4.1 For any axiom $\xi$, the following two sequent rules are equivalent

$$
\frac{\vdash \Sigma_{1} \quad \cdots \quad \vdash \Sigma_{n}}{\vdash \Gamma, \xi} \text { and } \frac{\vdash \Sigma_{1} \quad \cdots \quad \vdash \Sigma_{n} \vdash \Delta, \xi^{\perp}}{\vdash \Gamma, \Delta} \text {, }
$$

where $\Delta$ is fresh. If $\xi \in \mathcal{P}_{1}$, then the rules in (3) are equivalent to a rule

$$
\frac{\vdash \Sigma_{1} \quad \cdots \quad \vdash \Sigma_{n} \quad \vdash \Delta, A_{1,1}, \ldots, A_{1, k_{1}} \quad \cdots \quad \vdash \Delta, A_{m, 1}, \ldots, A_{m, k_{m}}}{\vdash \Gamma, \Delta}
$$

where each $A_{i, j}$ is atomic and $m, k_{1}, \ldots, k_{m} \geq 0$.

Proof The first equivalence is shown in one direction by letting $\Delta=\xi$ and using the ax-rule and in the other direction by using cut. If $\xi \in \mathcal{P}_{1}$ then $\xi^{\perp} \in \mathcal{N}_{1}$, and hence $\xi^{\perp}$ is equivalent to $\&_{i}\left(\mho_{j} A_{i, j}\right)$. Then (4) follows by using the (invertible) rules $8, \&, \top$ and $\perp$.

Theorem 4.2 Every $\mathcal{N}_{2}$-axiom can be transformed into a finite set of equivalent structural sequent rules, whose conclusions consist only of multiset variables. 
Proof Let $\phi$ be any $\mathcal{N}_{2}$-axiom. By Proposition $3.3 \phi$ is equivalent to a finite set $\left\{\psi_{1}, \ldots, \psi_{n}\right\}$ of $\mathcal{N}_{2}$-normal axioms, which means that each $\psi_{i}$ is equivalent to

$$
\overline{\vdash \xi_{i, 1}, \ldots, \xi_{i, m_{i}}}
$$

where each $\xi_{i, j} \in \mathcal{P}_{1}$. The claim follows by repeatedly applying Lemma 4.1.

Example 4.3 Applying the above procedure to excluded middle and Nelson axiom respectively (see Figure 3 ) yields the structural rules:

$\mathrm{em}^{\prime} \frac{\vdash \Gamma, A \quad \vdash \Gamma, A^{\perp}}{\vdash \Gamma} \quad \mathrm{nel}^{\prime} \frac{\vdash \Gamma, A^{\perp}, A^{\perp}, B \quad \vdash \Gamma, A^{\perp}, B, B \quad \vdash \Delta, A \quad \vdash \Sigma, B^{\perp}}{\vdash \Gamma, \Delta, \Sigma}$

These rules will be further transformed in Section 6 into equivalent rules obeying the subformula property.

By suitably adapting the procedure above we show below how to generate logical rules for connectives which are defined via $\mathcal{N}_{2}$ axioms.

Theorem 4.4 Let $\circledast$ be a connective. Any axiom of the shape $\phi \ngtr(A \circledast B)$, where $A$ and $B$ are formula variables and $\phi \in \mathcal{N}_{2}$, is equivalent to a finite set of logical sequent rules for $\circledast$.

Proof By Proposition $3.3 \phi \ngtr(A \circledast B)$ is equivalent to a finite set $\left\{\psi_{1} \diamond(A \circledast\right.$ $B), \ldots, \psi_{n}$ \& $\left.(A \circledast B)\right\}$ where each $\psi_{i}$ is $\mathcal{N}_{2}$-normal. Hence each $\psi_{i}$ \& $(A \circledast B)$ is equivalent to

$$
\overline{\vdash \xi_{i, 1}, \ldots, \xi_{i, m_{i}}, A \circledast B}
$$

where each $\xi_{i, j} \in \mathcal{P}_{1}$. By repeatedly applying Lemma 4.1 we eliminate all $\xi_{i, j}$ thus obtaining a logical rule for $A \circledast B$.

Note that Theorem 4.4 also applies for $n$-ary connectives. In Section 7 we show two examples of the usage of this theorem for the binary case.

\section{From $\mathcal{P}_{3}^{\prime}$-axioms to Hypersequent Rules}

In this section we show how to obtain structural rules in hypersequent calculus which are equivalent to $\mathcal{P}_{3}^{\prime}$-axioms ( $\mathcal{P}_{3}$, in presence of weakening). Our procedure generalizes the one in [4].

First notice that Lemma 4.1 can be extended to hypersequent calculus, without having to change the proof (by using ew and ec), as follows:

Lemma 5.1 For any axiom $\xi$, the following hypersequent rules are equivalent

$$
\frac{\mathscr{G}_{1} \ldots \quad \mathscr{G}_{n}}{\mathscr{H}\left|\mathscr{H}^{\prime}\right|+\Gamma, \xi} \quad \text { and } \quad \frac{\mathscr{G}_{1} \quad \cdots \quad \mathscr{G}_{n} \quad \mathscr{H} \mid+\Delta, \xi^{\perp}}{\mathscr{H}\left|\mathscr{H}^{\prime}\right|+\Gamma, \Delta},
$$

where $\Delta$ is fresh. If $\xi \in \mathcal{P}_{1}$, then the rules in (7) are equivalent to a rule

$$
\frac{\mathscr{G}_{1} \quad \ldots \quad \mathscr{G}_{n} \quad \mathscr{H}\left|\vdash \Delta, A_{1,1}, \ldots, A_{1, k_{1}} \quad \cdots \quad \mathscr{H}\right| \vdash \Delta, A_{m, 1}, \ldots, A_{m, k_{m}}}{\mathscr{H}\left|\mathscr{H}^{\prime}\right| \vdash \Gamma, \Delta},
$$

where each $A_{i, j}$ is atomic and $m, k_{1}, \ldots, k_{m} \geq 0$. 
Definition 5.2 A hypersequent structural rule or hyperstructural rule is

$$
\frac{\mathscr{H}\left|\vdash \Psi_{1} \quad \ldots \quad \mathscr{H}\right| \vdash \Psi_{n}}{\mathscr{H}\left|\vdash \Phi_{1}\right| \ldots \mid \vdash \Phi_{m}}
$$

where each $\Phi_{i}$ and $\Psi_{j}$ contains only multiset variables and formula variables.

Theorem 5.3 Every $\mathcal{P}_{3}^{\prime}$-axiom is equivalent to a finite set of hyperstructural rules where $\Phi_{1}, \ldots, \Phi_{n}$ consist of mutually distinct multiset variables.

Proof Let $\phi$ be a $\mathcal{P}_{3}^{\prime}$-axiom. By Proposition 3.6, $\phi$ is equivalent to a finite set $\left\{\psi_{1}, \ldots, \psi_{n}\right\}$ of formulas such that $\psi_{i}=\bigoplus_{j}\left(\chi_{i, j}\right)_{\& 1}$ where $\chi_{i, j}$ is $\mathcal{N}_{2}$-normal for all $i, j$. Thus, by Proposition 2.2 (see Def. 2.1 and 3.4), each $\psi_{i}$ is equivalent to

$$
\vdash \xi_{i, 1,1}, \ldots, \xi_{i, 1, m_{i 1}}|\ldots| \ldots \mid \vdash \xi_{i, k, 1}, \ldots, \xi_{i, k, m_{i k}}
$$

where each $\xi_{i, j, l} \in \mathcal{P}_{1}$. By presence of the ew-rule, (10) is equivalent to

$$
\overline{\mathscr{H}\left|\vdash \xi_{i, 1,1}, \ldots, \xi_{i, 1, m_{i 1}}\right| \ldots|\ldots| \vdash \xi_{i, k, 1}, \ldots, \xi_{i, k, m_{i k}}}
$$

Thus, to each component of (11) we can apply the same procedure as in the proof of Theorem 4.2, by using Lemma 5.1 instead of Lemma 4.1.

Corollary 5.4 Every $\mathcal{P}_{3}$-axiom is equivalent to a finite set of hyperstructural rules in presence of weakening.

Proof This follows from Theorem 5.3 and the fact that $\vdash_{\text {MALL }+\mathrm{w}} A \circ \cdots A \& 1$.

Example 5.5 The axiom $A \oplus\left(A^{\perp}\right)^{\ngtr n}$ in Figure 3 ( $n$-excluded middle) is equivalent to the following structural rule, in the presence of weakening

$$
n-\mathrm{em}^{\prime} \frac{\mathscr{H}\left|\vdash \Sigma_{1}, A^{\perp} \quad \ldots \quad \mathscr{H}\right| \vdash \Sigma_{n}, A^{\perp} \quad \mathscr{H} \mid \vdash \Gamma, A}{\mathscr{H}|\vdash \Gamma| \vdash \Sigma_{1}, \ldots, \Sigma_{n}}
$$

Remark 5.6 The step from Theorem 4.2 to Theorem 4.4 can also be done in the setting of hypersequents. Indeed, suppose that a connective $\circledast$ appears in an axiom $\phi$ which is equivalent to a set of rules of the shape

$$
\overline{\vdash \xi_{1,1}, \ldots, \xi_{1, m_{1}}|\ldots| \ldots \mid \vdash \xi_{k, 1}, \ldots, \xi_{k, m_{k}}, A \circledast B}
$$

We can apply the same procedure as in the proof of Theorem 5.3 to obtain a set of logical hypersequent rules for $\circledast$.

\section{Rule Completion and Cut-elimination}

Let us take stock of what we achieved so far. Sections 4 and 5 contain procedures to transform axioms up to the class $\mathcal{P}_{3}^{\prime}\left(\mathcal{P}_{3}\right.$, in presence of w) into equivalent (hyper)structural rules. Here we show how they can, provided they are acyclic, be transformed into equivalent rules which preserve cut-elimination when added to HMALL. A uniform and constructive cut elimination proof for HMALL extended with these rules is presented. 
Definition 6.1 The cut-closure CUT( $r$ ) of a (hyper)structural rule $r$ is the minimal set which contains the premises of $r$ and it is closed under applications of the cut rule. A rule $\mathrm{r}$ is said to be cyclic if for some formula variable $A$, we have $\mathscr{H} \mid \vdash \Gamma, A, A^{\perp} \in \mathrm{CUT}(\mathrm{r})$. Otherwise $\mathrm{r}$ is acyclic.

Example 6.2 The rules $\mathrm{em}^{\prime}$, nel', and $n$-em' in Examples 4.3 and 5.5 are acyclic. On the other hand, the following two rules are cyclic:

$$
\text { cancel } \frac{\vdash \Gamma, A, A^{\perp}}{\vdash \Gamma} \quad \text { and } \quad \text { menace } \frac{\vdash \Gamma, A^{\perp}, A^{\perp} \quad \vdash \Delta, A, A}{\vdash \Gamma, \Delta}
$$

Definition 6.3 We call a hyperstructural rule $r$ completed if it satisfies the following properties [4]:

- No Formula Variable (NFV): The conclusion and all premises of $\mathbf{r}$ contain only multiset variables and hypersequent contexts.

- Linear Conclusion $(L C)$ : Each multiset variable occurs at most once in the conclusion of $r$.

- Subformula Property (SP): Each multiset variable occurring in the premises of $r$ also occurs in the conclusion.

The conditions (NFV) and (LC) are crucial for the cut-elimination proof below (see, e.g., [24] for counterexamples when either of them is violated). Condition (SP) ensures that cut-elimination implies the subformula property. The rules generated by our procedures satisfy (NFV) for the conclusion, and (LC) and (SP) for multiset variables. Thus, for transforming them into equivalent completed rules it is enough to remove formula variables from the premises. This is done in the proof of the following theorem, by suitably modifying the "cutting step" of [4].

Theorem 6.4 Any acyclic (hyper)structural rule $\mathbf{r}$ generated by the procedures in Theorems 4.2 and 5.3 can be transformed into an equivalent completed rule.

Proof By induction on the number of formula variables in the premises of $r$. Let $A$ be one such variable. We denote by $\mathcal{G}_{A}^{+}$and $\mathcal{G}_{A}^{-}$the (subsets of the) premises of $\mathrm{r}$ which contain at least one occurrence of $A$ and $A^{\perp}$, respectively. If $\mathcal{G}_{A}^{+}=\emptyset$ we remove $\mathcal{G}_{A}^{-}$. As $A$ does not appear in the conclusion of $\mathrm{r}$, the resulting rule implies the original one by instantiating $A$ with $T$. The case $\mathcal{G}_{A}^{-}=\emptyset$ is similar. Otherwise, note that $A^{\perp} \notin \mathcal{G}_{A}^{+}$and $A \notin \mathcal{G}_{A}^{-}$by acyclicity; moreover if some hypersequent in $\mathcal{G}_{A}^{+}$(resp. $\mathcal{G}_{A}^{-}$) contains several occurrences of $A$ (resp. of $A^{\perp}$ ) then no hypersequent in $\mathcal{G}_{A}^{-}$(resp. in $\mathcal{G}_{A}^{+}$) contains more than one occurrence of $A^{\perp}$ (resp. of $A$ ). Hence we may assume w.l.o.g. that $\mathcal{G}_{A}^{+}=\left\{\mathscr{H} \mid \vdash \Upsilon_{i}, A: 1 \leq i \leq m\right\}$ and $\mathcal{G}_{A}^{-}=\left\{\mathscr{H} \mid \vdash \Phi_{k}, A^{\perp}, \ldots, A^{\perp}: 1 \leq k \leq n\right\}$. Let $\mathcal{G}_{A}^{\text {cut }}=\left\{\mathscr{H} \mid+\Phi_{k}, \Upsilon_{i_{1}}, \ldots, \Upsilon_{i_{k}}: 1 \leq k \leq n\right.$ and $\left.1 \leq i_{1}, \ldots, i_{k} \leq m\right\}$. Let $\mathbf{r}^{\prime}$ be the rule obtained by replacing in $r$ the premises $\mathcal{G}_{A}^{-} \cup \mathcal{G}_{A}^{+}$with $\mathcal{G}_{A}^{\text {cut }}$. We show that $r^{\prime}$ is equivalent to $r$. The direction $r^{\prime} \Rightarrow r$ easily follows by using cut. For the other direction, we set $\tilde{\tilde{A}}=\bigoplus_{i=1}^{m} \Upsilon_{i}$. Clearly $\vdash_{\mathrm{HMALL}} \mathscr{H} \mid \vdash \Upsilon_{i}, \tilde{A}$, for all $i=1, \ldots, m$, and for each $k=1, \ldots, n$, the hypersequent $\mathscr{H} \mid \vdash \Phi_{k}, \tilde{A}^{\perp}, \ldots, \tilde{A}^{\perp}$ is derivable from $\mathcal{G}_{A}^{\text {cut }}$ using the \&-rule. By applying $r$ we get the conclusion of $r^{\prime}$. Acyclicity is preserved, the number of formula variables decreased by one. 
Example 6.5 By applying the procedure in Theorem 6.4 to the rules in Examples 4.3 and 5.5 we obtain the equivalent completed rules em, nel and $n$-em of Figure 4. MALL + nel is the cut-free calculus recently introduced in [12] for constructive logic with strong negation (also known as Nelson's logic). HMALL $+\mathrm{w}+n$-em is instead a cut-free calculus for MALL extended with weakening and $n$-excluded middle (see Figure 3). The latter logic coincides with 3-valued Łukasiewicz logic when $n=2$ and with the logic IMT3 of [5] for $n=3$.

Conjecture 6.6 In presence of $\mathrm{w}$, any (hyper) structural rule generated by Theorems 4.2 and 5.3 can be transformed into an equivalent completed rule.

The construction of the completed rule proceeds similarly as in the proof of Theorem 6.4: Let $\hat{\mathcal{G}}_{A}$ be the cut-closure of $\mathcal{G}_{A}^{+} \cup \mathcal{G}_{A}^{-}$with cut-formula $A$ and without $\mathcal{G}_{A}^{+} \cup \mathcal{G}_{A}^{-}$, and let $\downarrow \hat{\mathcal{G}}_{A}$ be the set of minimal elements of $\hat{\mathcal{G}}_{A}$ wrt. the application of w. Note that if $\mathrm{r}$ is cyclic, then $\hat{\mathcal{G}}_{A}$ is infinite, but $\downarrow \hat{\mathcal{G}}_{A}$ is finite. Let $\downarrow \hat{\mathcal{G}}_{A}^{+}$be the set of hypersequents in $\downarrow \hat{\mathcal{G}}_{A}$ that do not contain $A^{\perp}$, and let $\mathcal{G}_{A}^{\text {cut }}$ be obtained from $\downarrow \hat{\mathcal{G}}_{A}^{+}$by deleting $A$ everywhere. Let $\mathrm{r}^{\prime}$ and $\mathrm{r}^{\prime \prime}$ be the rules obtained from $\mathrm{r}$ by replacing the premises $\mathcal{G}_{A}^{+} \cup \mathcal{G}_{A}^{-}$with $\downarrow \hat{\mathcal{G}}_{A}$ and $\mathcal{G}_{A}^{\text {cut }}$, respectively. Then it remains to show that $r, r^{\prime}$, and $r^{\prime \prime}$ are equivalent. (Note that $r^{\prime} \Rightarrow r^{\prime \prime}$ follows by setting $A=\perp$ and $A^{\perp}=1$ since 1 behaves as $\top$ in the presence of w.)

Example 6.7 By applying the procedure sketched above to menace, we get the contraction rule c, while cancel yields the (contradictory) rule $\frac{}{\vdash \Gamma}$. It is easy to see that the obtained rules are equivalent to menace and cancel, respectively.

Let us write HMALL ${ }^{\text {ext }}$ to denote HMALL extended with any set of completed rules. We now outline a syntactic proof of cut-elimination for HMALL ${ }^{\text {ext }}$ (see [4] for a semantic proof in the single-conclusion setting). As usual, the length $|d|$ of a (hyper)sequent derivation $d$ is the maximal number of inference rules +1 occurring on any branch of $d$. The complexity $|A|$ of a formula $A$ is the number of occurrences of its connectives. The cut rank $\rho(d)$ of $d$ is the maximal complexity of the cut-formulas in $d$ plus 1 . Clearly $\rho(d)=0$ if $d$ has no cuts.

Lemma 6.8 Let $d_{+}$and $d_{-}$be derivations in $\mathrm{HMALL}{ }^{\mathrm{ext}}$ such that

(i) $d_{+}$is a derivation of $\mathscr{H} \mid \vdash \Gamma, A$ and $\rho\left(d_{+}\right) \leq|A|$, and

(ii) $d_{-}$is a derivation of $\mathscr{H} \mid \vdash \Sigma, A^{\perp}$ and $\rho\left(d_{-}\right) \leq|A|$, and

(iii) $A$ is a compound formula and one of $d_{+}$or $d_{-}$ends with a logical rule introducing A.

Then we can find a derivation $d$ in $\mathrm{HMALL}^{\mathrm{ext}}$ of $\mathscr{H} \mid \vdash \Gamma, \Sigma$ with $\rho(d) \leq|A|$.

Of course, one could derive $\mathscr{H} \mid \vdash \Gamma, \Sigma$ by applying cut, but the resulting derivation would then have cut-rank $|A|+1$.

Proof Assume w.l.o.g. that $d_{-}$ends with a logical rule introducing $A^{\perp}$. Consider a derivation $d_{+}^{\prime}$ of $\mathscr{H}\left|\vdash \Gamma_{1}, A^{\lambda_{1}}\right| \ldots \mid \vdash \Gamma_{n}, A^{\lambda_{n}}$ with $\rho\left(d_{+}^{\prime}\right) \leq|A|$ where $A^{\lambda}$ stands for $A, \ldots, A$ ( $\lambda$ times). We prove, by induction on $\left|d_{+}^{\prime}\right|$, that one can find a derivation of $\mathscr{H}\left|\vdash \Gamma_{1}, \Sigma^{\lambda_{1}}\right| \ldots \mid \vdash \Gamma_{n}, \Sigma^{\lambda_{n}}$ with cut-rank $\leq|A|$. This is required 
to deal with internal and external contraction rules. If $d_{+}^{\prime}$ ends in an axiom $(\mathrm{ax}, 1, \top)$ then we are done. Otherwise, let $\mathrm{r}$ be the last inference rule in $d_{+}^{\prime}$.

(a) If $r$ acts only on $\mathscr{H}$ or $r$ is ew, ec or $\perp$ then the claim follows by the induction hypothesis and an application of $r$. The same holds when $r$ is a logical rule which does not introduce a cut formula $A$.

(b) Suppose that $r$ is an introduction rule for $A$. The claim easily follows by applying cut to the premise(s) of $r$ and to the premise(s) of the last rule applied in $d_{-}$(which is a logical rule introducing $A^{\perp}$ ). The cut-formula(s) of the newly introduced cut is (are) the auxiliary formula(s) of $A$ and therefore the resulting derivation has cut-rank $\leq|A|$.

(c) If $r$ is any completed rule then the properties of (NFV) and (LC) allow the cut to be shifted upward over the rule premises. The claim follows by the induction hypothesis and an application of $r$.

Lemma 6.9 Let $d_{+}$and $d_{-}$be derivations in $\mathrm{HMALL}^{\mathrm{ext}}$ such that the hypothesis (i) and (ii) of Lemma 6.8 hold. Then we can find a derivation d in HMALL ${ }^{\text {ext }}$ of $\mathscr{H} \mid \vdash \Gamma, \Sigma$ with $\rho(d) \leq|A|$.

Proof Proceed similarly to that of Lemma 6.8. If the last inference rule applied is any rule other than a logical rule introducing a cut-formula $A$, the proof proceeds as in cases (a) and (c). Otherwise the claim follows by induction hypothesis, an application of $r$ and Lemma 6.8.

Theorem 6.10 (Cut-elimination) HMALL extended with any set of completed rules admits cut-elimination.

Proof Let $d$ be a derivation in HMALL ext with $\rho(d)>0$. The proof proceeds by a double induction on $(\rho(d), \# \rho(d))$, where $\# \rho(d)$ is the number of cuts in $d$ with cut-rank $\rho(d)$. Consider an uppermost application of cut in $d$ with cut-rank $\rho(d)$. By applying Lemma 6.9 to its premises either $\rho(d)$ or $\# \rho(d)$ decreases.

Remark 6.11 Let $S$ be any set of hypersequents that (1) contain only atomic formulas, (2) are closed under cut, and (3) do not contain any hypersequent of the form $\mathscr{H} \mid \vdash \Gamma, A, A^{\perp}$. Then our cut-elimination proof also allows the elimination of cuts from HMALL ext proofs whose leaves are either axioms (ax, $1, \top$ ) or hypersequents belonging to $S$. This establishes a stronger form of cut-elimination.

\section{A Case Study: Abelian and Łukasiewicz Logics}

Consider the axiom inv: $A \otimes(A \multimap 1)$. Writing $A^{-1}$ for $A \multimap 1$ and noting that inv is equivalent to $1 \multimap \cdots\left(A \otimes A^{-1}\right)$, one immediately sees that inv states that $A^{-1}$ is the inverse element of $A$ with unit 1. Adding inv to IMALL yields a contradiction as $\vdash_{\text {IMALL }} 0 \otimes 0^{-1} \rightarrow A$. However, IMALL + inv without $T$ and 0 is consistent. Indeed, the logic $A L=I M A L L \backslash\{\top, 0, \perp\}+$ inv has the lattice ordered Abelian groups as models (see, e.g., [18]), and for this reason is called Abelian logic [15, $16]$.

Observe that inv $\in \mathcal{P}_{3}$, but unfortunately inv $\notin \mathcal{P}_{3}^{\prime}$. Hence the method developed in [4] does not apply. What can we do? 
Episode 1: The Power of Multiple Conclusion. First, we move to the classical setting. Let MALL ${ }^{-}$denote MALL without $T$ and 0 . In $\mathrm{MALL}^{-}$, the

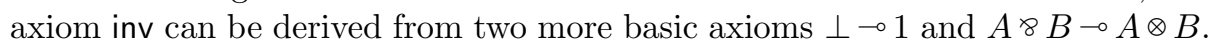
that we call mixax and mixinv, respectively. The interesting observation is that these two axioms are not only sufficient, but also necessary:

Theorem 7.1 For every AL-formula A, we have that $\mathrm{AL}$ proves $A$ if and only if $\mathrm{MALL}^{-}+$mixax + mixinv proves $A$.

Proof $(\Rightarrow)$ Observe that $\mathrm{MALL}^{-}+$mixax + mixinv proves inv. $(\Leftarrow)$ Prove by induction that if $\mathrm{MALL}^{-}+\operatorname{mixax}+$ mixinv proves $\vdash A_{1}, \ldots, A_{n}$, then $\vdash_{\mathrm{AL}} \otimes_{i} A_{i}^{a}$, where $A_{i}^{a}$ is obtained from $A_{i}$ by replacing $\perp$ by 1 , ४ by $\otimes$, and $a^{\perp}$ by $a \multimap 1$.

There are two important observations to make: First, mixax and mixinv are both in $\mathcal{N}_{2}$ and therefore Theorem 4.2 applies. Second, mixinv is not an intuitionistic axiom. Thus, the shift to the multiple conclusion setting is crucial.

Episode 2: The Structuralization of the Axioms. Let us now apply Theorem 4.2 to produce structural rules equivalent to mixax and mixinv:

$$
\begin{aligned}
& \operatorname{mixax}: \perp \multimap 1 \leadsto \overline{\vdash 1,1} \leadsto \frac{\vdash \Gamma, \perp \vdash \Delta, \perp}{\vdash \Gamma, \Delta} \leadsto \operatorname{mix} \frac{\vdash \Gamma \vdash \Delta}{\vdash \Gamma, \Delta} \\
& \text { mixinv : } A \ngtr B-A \otimes B \leadsto \frac{}{\vdash A^{\perp} \otimes B^{\perp}, A \otimes B} \leadsto \frac{\vdash \Gamma, A^{\perp}, B^{\perp} \vdash \Delta, A, B}{\vdash \Gamma, \Delta}
\end{aligned}
$$

The result for mixinv is a cyclic rule. One formula variable (e.g. $B$ ) can be removed using the procedure in the proof of Theorem 6.4 thus obtaining the cancel rule of Example 6.2. However, there is no way to proceed further to obtain any equivalent completed rule.

Episode 3: Why Not Logical Rules? Instead of transforming mixinv into a structural rule, let us apply Theorem 4.4 to obtain a new logical rule for the $\otimes$-connective. Indeed:

$$
\text { mixinv : } \quad A \ngtr B \multimap A \otimes B \quad \leadsto \frac{}{\vdash A^{\perp} \otimes B^{\perp}, A \otimes B} \leadsto \otimes^{\prime} \frac{\vdash \Gamma, A, B}{\vdash \Gamma, A \otimes B}
$$

The rule $\otimes$ is then derivable from mix and $\otimes^{\prime}$, and can be removed. We now have a system in which only the cut rule does not have the subformula property. However, every attempt to eliminate cut will introduce the cancel rule, and every attempt to eliminate the cancel rule will introduce cut - a dead end.

Episode 4: A New Hope. By inspecting the failed attempts for cut/cancelelimination, we find a concrete counterexample, that is the excluded middle law $A \oplus A^{\perp}$. It belongs to $\mathcal{P}_{1}$, so why not transforming it into a structural rule? By applying our procedure, we obtain the equivalent rule em in Figure 4. Hence we can safely add em without changing the logical strength of the system. Do we now have cut and cancel elimination? 
Episode 5: Counterexample Strikes Back. Unfortunately, no. We find two more counterexamples: $\perp$ and $(A \& 1) \oplus\left(A^{\perp} \& 1\right)$. The former is equivalent to the nullary mix rule mix $_{0} \frac{-}{\vdash}$, while the latter, which we call splax, is in $\mathcal{P}_{3}^{\prime}$. We can therefore apply Theorems 5.3 and 6.4 to obtain a hyperstructural rule:

$$
\text { splax } \leadsto \frac{}{\mathscr{H}|\vdash A| \vdash A^{\perp}} \leadsto \frac{\mathscr{H}\left|\vdash \Gamma, A^{\perp} \mathscr{H}\right| \vdash \Delta, A}{\mathscr{H}|\vdash \Gamma| \vdash \Delta} \leadsto \text { split } \frac{\mathscr{H} \mid \vdash \Gamma, \Delta}{\mathscr{H}|\vdash \Gamma| \vdash \Delta}
$$

This leads us to switch from $\mathrm{MALL}^{-}$to its hypersequent counterpart $\mathrm{HMALL}^{-}$, and accordingly generalize mix and $\otimes^{\prime}$ to their hypersequent counterparts, which we still call mix and $\otimes^{\prime}$. A good news is that the previous rule em is redundant in presence of split.

Episode 6: The Return of Cut Elimination. We have finally arrived at the system $\mathrm{HAL}=\mathrm{HMALL}^{-}+\mathrm{mix}+\mathrm{mix}_{0}+\otimes^{\prime}+$ split for AL introduced in [14]. A concrete cut-elimination procedure which relies on the invertibility of logical rules is contained in [13]. Hence, we have

Theorem 7.2 HAL admits cut-elimination.

The General Pattern. Our development so far suggests the following heuristics to find a cut-free calculus for a given logic.

1. Convert axioms into structural/logical rules having the subformula property.

2. If we obtain a cut-free system, we are done. Otherwise, find a counterexample $A$ by inspecting the failure of cut-elimination.

3. If $A \in \mathcal{N}_{2}$ or $A \in \mathcal{P}_{3}^{\prime}$, apply Theorem 4.2 or 5.3 accordingly and go to 2 . (Otherwise, we get stuck.)

One can think of it analogous to the Knuth-Bendix algorithm for obtaining a confluent rewriting system out of a set of equations. But the analogy is only shallow, since ours is neither complete nor gives rise to a semi-decision procedure.

Another Example. A similar situation arises for infinite-valued Łukasiewicz

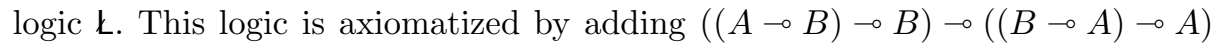
to IMALL. In $k$, the additive disjunction is definable from linear implication: $((A \multimap B) \multimap B) \circ \cdots A \oplus B$. Hence the above axiom just states the commutativity of $\oplus$. The axiom is in $\mathcal{N}_{3}$, so cannot be dealt with by our general method.

Episode 7: Defining a New Connective. It is known that $\ell$ can be faithfully interpreted in Abelian logic. In particular, the Łukasiewicz implication $A \stackrel{ \pm}{\Rightarrow} B$ can be defined by $(A \stackrel{\mathrm{L}}{\Rightarrow} B) \circ(A \multimap B)_{\& 1}$ inside Abelian logic [14]. Now, each of the two directions yields logical rules via Theorem 4.4 (and the equivalences $1 \odot \cdots \perp, A \otimes B \circ \cdots A \ngtr B)$ : one for $A \stackrel{\Xi}{\Rightarrow} B$, and two for its DeMorgan dual $A \stackrel{\Sigma}{\longleftarrow} B:^{2}$

$$
\stackrel{\llcorner}{\Rightarrow} \frac{\vdash \Gamma, A^{\perp}, B \quad \vdash \Gamma}{\vdash \Gamma, A \stackrel{\llcorner}{\Rightarrow} B} \quad \longleftarrow 上_{1} \frac{\vdash \Gamma}{\vdash \Gamma, A \longleftarrow B} \quad \longleftarrow_{2} \frac{\vdash \Gamma, A, B^{\perp}}{\vdash \Gamma, A \longleftarrow B}
$$

These are the implication rules of the cut-free calculus for $\measuredangle$ introduced in [14].

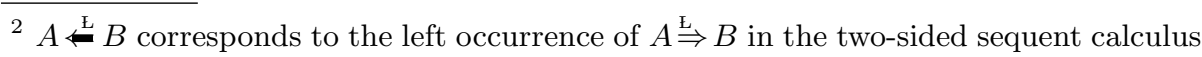
(cf. Remark 2.4), not to be confused with the Łukasiewicz negation of $A \stackrel{\unrhd}{\Rightarrow} B$. 


\section{Concluding Remarks}

Relation to Algebraic Completions. Given a (hyper)sequent calculus H, we denote by $\mathrm{H}^{\infty}$ its extension with infinitary conjunction $\&_{i \in I} A_{i}$ and disjunction $\bigoplus_{i \in I} A_{i}$, where $I$ is an arbitrary index set, together with suitable logical rules generalizing \& and $\oplus$. We say that $\mathrm{H}^{\infty}$ is conservative over $\mathrm{H}$ if for any set $\mathcal{S} \cup\{A\}$ of $\mathrm{H}$-formulas, $\mathcal{S} \vdash_{\mathrm{H}^{\infty}} A$ implies $\mathcal{S} \vdash_{\mathrm{H}} A$.

Since cut-elimination is a canonical way to show conservativity, one can expect that if all rules of $\mathrm{H}$ are "good", i.e. admit a suitably strong form of cutelimination, then $\mathrm{H}^{\infty}$ is conservative over $\mathrm{H}$. The work in [3] proves that any $\mathcal{N}_{2}$-axiom $\phi$ can be transformed into equivalent acyclic structural rules if and only if IMALL ${ }^{\infty}+\phi$ is conservative over IMALL $+\phi$. We conjecture that the same holds for $\mathcal{P}_{3}^{\prime}$ axioms, both in single and multiple-conclusion settings. Now the question is: do Abelian logic and Eukasiewicz logic admit such a strong form of cut-elimination which imply conservativity?

A negative answer arises from the following two facts:

(i) $\mathrm{H}^{\infty}$ is conservative over $\mathrm{H}$ if and only if the class $\mathfrak{V}(\mathrm{H})$ of algebras corresponding to $\mathrm{H}$ is closed under completions, in the sense that any $V \in \mathfrak{V}(\mathrm{H})$ can be embedded into a complete algebra in $\mathfrak{V}(\mathrm{H})$ [3, Prop. 5.9].

(ii) Both the class of lattice ordered Abelian groups and the class of MValgebras (the algebraic semantics of $\mathfrak{k}$ ) are not closed under completions, see, e.g., [11].

Therefore, though the calculi of [14] admit cut-elimination, their rules, which we extracted out of $\mathcal{P}_{3}$ and $\mathcal{N}_{3}$ axioms, are not "good" enough to ensure conservativity. This contrasts with the result for $\mathcal{N}_{2}$ axioms (and $\mathcal{P}_{3}^{\prime}$ ones, if our conjecture is true, cf. Remark 6.11).

Relation to Categories. In the category theoretical setting, Abelian logic lives in compact closed categories, whose canonical instance is the category of finite dimensional vector spaces over a fixed field. Indeed, $\mathrm{MALL}^{-} \backslash\left\{\&, \oplus_{1}, \oplus_{2}\right\}+$ mix $+\otimes^{\prime}$ is the calculus given by $[22]$ which aims to capture morphisms in a freely generated compact closed category via sequent proofs.

On the other hand, Abelian logic (and its hypersequent calculus HAL) also incorporates \& and $\oplus$, which in the the world of categories are usually interpreted as binary products and coproducts. Thus a natural question is whether there is a nicely behaved equivalence relation on proofs in $\mathrm{HAL}$ such that the equivalence classes are the morphisms in the free compact closed category with binary products and coproducts (as it is achieved by the rule permutations in the sequent calculus for multiplicative linear logic and star-autonomous categories [10]).

Two observations: First, if we add initial and terminal objects, and therefore get all finite products and coproducts, we get a collapse: products and coproducts coincide [6]. In terms of logic we have $A \& B \cong A \oplus B$, and therefore an inconsistency. This is not a surprise: we have seen above that adding 0 and $\top$ to AL makes the logic inconsistent. Second, sequents are category theoretically well studied in the form of polycategories [23], but it has not yet been investigated what hypersequents mean in terms of categories. 


\section{References}

1. J.-M. Andreoli. Logic programming with focusing proofs in linear logic. Journal of Logic and Computation, 2(3):297-347, 1992.

2. A. Avron. Hypersequents, logical consequence and intermediate logics for concurrency. Ann. Math. Artif. Intell., 4:225-248, 1991.

3. A. Ciabattoni, N. Galatos, and K. Terui. Algebraic proof theory for substructural logics: Cut-elimination and completions. Submitted, 2008.

4. A. Ciabattoni, N. Galatos, and K. Terui. From axioms to analytic rules in nonclassical logics. In LICS, pages 229-240, 2008.

5. J. Gispert and A. Torrens. Axiomatic extensions of IMT3 logic. Studia Logica, 81(3):311-324, 2005.

6. R. Houston. Finite products are biproducts in a compact closed category. Journal of Pure and Applied Algebra, 212(2), 2008.

7. M. Kracht. Power and weakness of the modal display calculus. In Proof theory of modal logic, pages 93-121. Kluwer, 1996.

8. F. Lamarche. On the algebra of structural contexts. Accepted at Mathematical Structures in Computer Science, 2001.

9. F. Lamarche and C. Retoré. Proof nets for the Lambek-calculus - an overview. In V. M. Abrusci and C. Casadio, editors, Proceedings of the Third Roma Workshop "Proofs and Linguistic Categories", pages 241-262. CLUEB, Bologna, 1996.

10. F. Lamarche and L. Straßburger. From proof nets to the free *-autonomous category. Logical Methods in Computer Science, 2(4:3):1-44, 2006.

11. T. Litak and T. Kowalski. Completions of GBL algebras: negative results. Algebra Universalis, 58:373-384, 2008.

12. G. Metcalfe. A sequent calculus for constructive logic with strong negation as a substructural logic. To appear in Bulletin of the Section of Logic.

13. G. Metcalfe. Proof theory for Casari's comparative logics. J. Log. Comput., 16(4):405-422, 2006.

14. G. Metcalfe, N. Olivetti, and D.M. Gabbay. Sequent and hypersequent calculi for Abelian and Łukasiewicz logics. ACM Trans. Comput. Log., 6(3):578-613, 2005.

15. R. Meyer and J. Slaney. Abelien logic (from A to Z). In Paraconsistent Logic: Essays on the Inconsistent, pages 245-288, 1989.

16. R. Meyer and J. Slaney. A, still adorable. In Paraconsistency: The Logical Way to the Inconsistent (Proceedings of the world congress on paraconsistency held in Sao Paulo), pages 241-260, 2002.

17. D. Miller. Forum: A multiple-conclusion specification logic. Theoretical Computer Science, 165:201-232, 1996.

18. T. Kowalski N. Galatos, P. Jipsen and H. Ono. Residuated Lattices: an algebraic glimpse at substructural logics. Elsevier, 2007.

19. S. Negri. Proof analysis in modal logics. Journal of Philosophical Logic, 34(56):507-544, 2005.

20. G. Sambin, G. Battilotti, and C. Faggian. Basic logic: Reflection, symmetry, visibility. J. Symb. Log., 65(3):979-1013, 2000.

21. H. Schellinx. Some syntactical observations on linear logic. Journal of Logic and Computation, 1(4):537-559, 1991.

22. M. Shirahata. A sequent calculus for compact closed categories. Unpublished, 2000 .

23. M. E. Szabo. Polycategories. Comm. Alg., 3:663-689, 1975.

24. K. Terui. Which structural rules admit cut elimination? An algebraic criterion. Journal of Symbolic Logic, 72(3):738-754, 2007. 